\title{
Possible hospital acquired Acinetobacter, Klebsiella, Mycoplasma and Legionella secondary infections in Covid19 patients from Russia Sandeep Chakraborty ${ }^{1}$,
}

\section{Letter}

The hypothesis that SARS-Cov2 enables anaerobic bacteria to colonize the lungs disrupting homeostasis, causing long-drawn chronic symptoms, and acute severe symptoms (ARDS, septic shock, clots, arterial stroke) which finds resonance, with key differences, in the 'forgotten disease' Lemierre Syndrome [1], enabled by Epstein Barr Virus [2] has found corroboration in other studies. An assessment of immunity in mild versus severe COVID-19 infection in humans found that severity correlated with increased bacterial products in plasma [3], while another study reported endotoxemia and circulating bacteriome in severe COVID-19 patients [4]. Fusobacterium nucleatum bacteremia was reported in Covid19 patients in Belgium [5]. The overlapping symptoms can often confuse diagnosis $[6,7]$. 'Jugular vein distention' should have led to a Lemierre like diagnosis [8].

While, these are commensal lung microbiome, hospital acquired infections (HAI) also play a major role in fatal outcomes for Covid19 patients.

\section{Secondary infections in samples from Russia}

Acinetobacter/Legionella/Mycoplasma/Klebsiella possible co-infections in Covid19 patients from Russia (Accid:PRJNA682735). Some of these samples have no SARS-Cov2 reads - these could be control samples. The viral load is quite low in many patients, indicating that these might lead to false negative results [9]. It is interesting to note that samples with higher viral load also have higher $\%$ of anaerobic bacteria.

Table 1: Acinetobacter/Legionella/Mycoplasma/Klebsiella co-infections in Covid19 patients from Russia (Accid:PRJNA682735): The viral load is quite low in all but four samples. Reads are per $100 \mathrm{k}$.

\begin{tabular}{|c|c|c|}
\hline Accid & Bacterial & Cov2 \\
\hline SRR13300913 & Acinetobacter=26320 HOMO=621 Legionella=253 & 0 \\
SRR13300932 & Stenotrophomonas=10329 Pseudomonas=5139 Acinetobacter=4162 & 0 \\
SRR13300811 & Acinetobacter=21025 Klebsiella=1549 Citrobacter=310 & 0 \\
SRR13300860 & Neisseria=4635 HOMO=3607 Streptococcus=3172 & 0 \\
\hline SRR13300797 & Acinetobacter=27550 HOMO=1546 Moraxella=290 & 1 \\
SRR13300858 & Acinetobacter=17845 HOMO=2530 Moraxella=78 & 1 \\
SRR13300898 & Acinetobacter=15613 Klebsiella=645 Citrobacter=148 & 1 \\
SRR13300909 & Acinetobacter=7677 Klebsiella=2300 Mycoplasma=1625 & 1 \\
SRR13300918 & Acinetobacter=13441 Klebsiella=1374 Raoultella=274 & 1 \\
SRR13300947 & Acinetobacter=27400 Klebsiella=1243 Legionella=300 & 1 \\
SRR13300878 & Klebsiella=8925 Raoultella=1691 Enterobacter $=1599$ & 4 \\
SRR13300802 & Mycoplasma=1538 Prevotella=936 Treponema=721 & 32 \\
SRR13300884 & Streptococcus=4542 Prevotella=1376 Mycoplasma=1229 & 76 \\
SRR13300914 & Streptococcus=8093 Prevotella=2339 Haemophilus=1001 & 466 \\
\hline
\end{tabular}




\section{Previous Acinetobacter/Legionella/Klebsiella/Mycoplasma co-infections in Covid19 patients}

Ventilator-associated pneumonia (VAP) caused by carbapenem-resistant Acinetobacter baumannii (CRAB) was a source of concern [10].

An acute care hospital in New Jersey saw an Increase in hospital-acquired CRAB infection and colonization [11]. In another example, all 19 patients were found positive for bacterial infections (17 Acinetobacter baumannii and 2 Staphylococcus aureus) [12]. There have been isolated incidents of Legionella and SARSCoV-2 co-infection, one of which led to a fatal result in an 80 year old patient returning from a Nile cruise [13]. In another case, which made a full recovery with antibiotics, CSF was PCR positive for Neisseria meningitidis [14]. Klebsiella pneumoniae infections were reported in an Italian hospital [15], while fatal sepsis hypervirulent Klebsiella pneumoniae was reported in a Japanese Covid19 patient [16] A concurrent infection with Klebsiella pneumoniae in Taiwan also led to a fatal outcome [17].

\section{References}

1. Weesner CL, Cisek JE (1993) Lemierre syndrome: the forgotten disease. Annals of emergency medicine 22: $256-258$.

2. Chakraborty S (2020). Sars-cov2 enables anaerobic bacteria (Prevotella, et al) to colonize the lungs disrupting homeostasis, causing long-drawn chronic symptoms, and acute severe symptoms (ARDS, septic shock, clots, arterial stroke) which finds resonance, with key differences, in the 'forgotten disease' Lemierre syndrome, enabled by Epstein Barr Virus. doi:10.31219/osf.io/usztn. URL osf .io/usztn.

3. Arunachalam PS, Wimmers F, Mok CKP, Perera RA, Scott M, et al. (2020) Systems biological assessment of immunity to mild versus severe COVID-19 infection in humans. Science 369: 12101220.

4. Sirivongrangson P, Kulvichit W, Payungporn S, Pisitkun T, Chindamporn A, et al. (2020) Endotoxemia and circulating bacteriome in severe COVID-19 patients. medRxiv .

5. Wolff L, Martiny D, Deyi VYM, Maillart E, Clevenbergh P, et al. (2020) COVID-19-associated Fusobacterium nucleatum bacteremia, Belgium. Emerging infectious diseases 27.

6. Repper DC, Arrieta AC, Cook JE, Renella P (2020) A case of Lemierre syndrome in the era of COVID-19: all that glitters is not gold. The Pediatric Infectious Disease Journal 39: e445-e447.

7. Howley F, O'Doherty L, McEniff N, O'Riordan R (2020) Late presentation of 'Lemierre's syndrome': how a delay in seeking healthcare and reduced access to routine services resulted in widely disseminated fusobacterium necrophorum infection during the global COVID-19 pandemic. BMJ Case Reports CP 13: e239269.

8. Dolhnikoff M, Ferranti JF, de Almeida Monteiro RA, Duarte-Neto AN, Gomes-Gouvêa MS, et al. (2020) SARS-Cov-2 in cardiac tissue of a child with COVID-19-related multisystem inflammatory syndrome. The Lancet Child \& Adolescent Health 4: 790-794.

9. Song L, Xiao G, Zhang X, Gao Z, Sun S, et al. (2020) A case of SARS-Cov-2 carrier for 32 days with several times false negative nucleic acid tests. medRxiv .

10. Lima WG, Brito JCM, da Cruz Nizer WS (2020) Ventilator-associated pneumonia (vap) caused by carbapenem-resistant Acinetobacter baumannii in patients with COVID-19: Two problems, one solution? Medical hypotheses 144: 110139. 
11. Perez S, Innes GK, Walters MS, Mehr J, Arias J, et al. (2020) Increase in hospital-acquired carbapenem-resistant Acinetobacter baumannii infection and colonization in an acute care hospital during a surge in COVID-19 admissions - new jersey, february-july 2020. Morbidity and Mortality Weekly Report 69: 1827.

12. Sharifipour E, Shams S, Esmkhani M, Khodadadi J, Fotouhi-Ardakani R, et al. (2020) Evaluation of bacterial co-infections of the respiratory tract in COVID-19 patients admitted to ICU. BMC infectious diseases 20: 1-7.

13. Arashiro T, Nakamura S, Asami T, Mikuni H, Fujiwara E, et al. (2020) Sars-cov-2 and legionella co-infection in a person returning from a nile cruise. Journal of Travel Medicine 27: taaa053.

14. Gallacher SD, Seaton A (2020) Meningococcal meningitis and covid-19 co-infection. BMJ Case Reports CP 13: e237366.

15. Arcari G, Raponi G, Sacco F, Bibbolino G, Di Lella FM, et al. (2020) Klebsiella pneumoniae infections in covid-19 patients: a two-month retrospective analysis in an italian hospital. International Journal of Antimicrobial Agents .

16. Hosoda T, Harada S, Okamoto K, Ishino S, Kaneko M, et al. (2020) Covid-19 and fatal sepsis caused by hypervirulent klebsiella pneumoniae, japan, 2020. Emerging Infectious Diseases 27.

17. Chen WC, Lai YC, Lin CH, Zheng JF, Hung WC, et al. (2020) First COVID-19 mortality case in Taiwan with bacterial co-infection by national surveillance of critically ill patients with influenzanegative pneumonia. Journal of Microbiology, Immunology and Infection . 